\title{
FLUCTUATIONS OF POLARIZED RADIATION IN A RANDOM MAGNETOPLASMA
}

\author{
ALEXANDER KUKUSHKIN \\ Ausralia Telescope National Facility, CSIRO, \\ PO Box 76,Epping NSW 2121 Australia
}

This paper analyses fourth-order moments of polarized radiation passing through magnetoactive plasma with random irregularities both in electron density and magnetic field. We consider the new propagation effect arising from chiral properties of random magnetoplasma. That is, the lenses formed by the same irregularities of magnetic field may be characterized by refractive properties of opposite sense vis-a-vis the rotation of wave polarization vector. This produces an appearance of slight circular polarization fluctuations arising from initially nonpolarised radiation. Analysis of the polarized radiation fluctuations may allow the spatial spectrum of magnetic field irregularities to be detected. The enchanced level of circularly polarized component, and the share of fluctuations owing to magnetic field irregularities, can be readily observed at low frequencies only, say in the radiation passing through the solar chromosphere or Jovian and terrestrial ionosphere (magnitosphere).

The shape of the wave amplitude or phase fluctuation spectrum in a plasma medium is relative to the spectrum of fluctuations in its refractive index, and hence that of electron density irregularities. In a number of cases question whether fluctuations in the external magnetic field also affect parameters of the radiation received at the observation point is of interest. Is it possible to restore parameters of the spatial spectrum of fluctuating magnetic field from the correlation function or power spectrum of observed electromagnetic scintillations? We believe that such measurements could be useful for the study of magnetogasdynamic turbulence in the polar ionosphere and magnetosphere and, in particular, near the magnetopause where the random magnetic field component is especially pronounced. The study of magnetic fields in cosmic sources of synhrotron radiation leads to a similar problem. Spangler's (1982) paper was dedicated to fluctuations of the linearly polarized radiation in a random magnetoplasma. The effect of the nonrelativistic electrons upon fluctuations of the $Q$ and $U$ Stokes' parameters was studied there on the basis of the radiation transfer equation which fails, however, to allow for diffraction effects. At centimeter wavelengths or in the case optically thin sources the method gives good results. At lower frequencies we obviously have to consider diffraction and, in particular include amplitude fluctuations in the analysis.

Consider the correlation functions of the Stokes parameters defined as

$$
\begin{aligned}
\Gamma_{\alpha} & =\Gamma_{\alpha}(\boldsymbol{\varrho}, \mathbf{R})= \\
& =\left\langle\tilde{S}_{\alpha}\left(\boldsymbol{\varrho}_{1}, \boldsymbol{\varrho}_{2}\right) * \tilde{S}_{\alpha}^{*}\left(\boldsymbol{\varrho}_{1}+\mathbf{R}, \boldsymbol{\varrho}_{2}+\mathbf{R}\right)\right\rangle-\left\langle\tilde{S}_{\alpha}\left(\boldsymbol{\varrho}_{1}, \boldsymbol{\varrho}_{2}\right)\right\rangle *\left\langle\tilde{S}_{\alpha}^{*}\left(\boldsymbol{\varrho}_{1}+\mathbf{R}, \boldsymbol{\varrho}_{2}+\mathbf{R}\right)\right\rangle
\end{aligned}
$$

where $\varrho=\varrho_{1}-\varrho_{2}$ and $S_{\alpha}$ is either of the components of the Stokes vector $\mathbf{S}=\{\tilde{I}, \tilde{Q}, \tilde{U}, \tilde{V}\}, \quad S_{\alpha}=S_{\alpha}\left(\varrho_{1}, \varrho_{2}\right)$.The latter represents a spatial Fourier com- 
ponent of the distribution $\mathbf{S}(\mathbf{n})$ of the polarized radiation from a source at the observation plane, $\mathbf{S}(\mathbf{n})=\{I(\mathbf{n}), Q(\mathbf{n}), U(\mathbf{n}, V(\mathbf{n})\}$ and let assume that initial Stokes vector to take the value $S_{0}(\mathbf{n})$.

The equations governing the correlation function of the Stokes parameters generally are very cumbersome. We will consider the case of coincident observation points ( i.e. $\varrho=0, \mathbf{R}=0$ ) and analyse the effect of both the electron density and magnetic field fluctuations. We shall also assume the source to be pointlike over the angular coordinates and the initial Stokes vector to take the value $\mathbf{S}_{0}=I_{0}, Q_{0}, U_{0}, V_{0}$. The fluctuations in the electron density and the magnetic field are statistically independent and both are of a turbulent nature. The spatial spectrum of the isotropic fluctuations can be written as

$$
\Phi_{N, H}(\kappa)=0.033 C_{N, H}{ }^{2} \kappa^{-11 / 3} \exp \left(-\kappa^{2} / \kappa_{m}^{2}\right)
$$

where $\kappa$ is a three- dimensional wave vector, $\kappa_{m}=5.92 / l_{0}, l_{0}=l_{0 N, H}$ are inner turbulent scales of the $\delta N$ and $\delta H$ irregularities, respectively, $C_{N, H}{ }^{2}$ are the structure constants of the relative fluctuations, $\delta H=(H-\langle H\rangle) /\langle H\rangle$. In Eq.(2) we have taken a Kolmogorov model for the spectra of $\delta N$ and $\delta H$, with a power index of $11 / 3$. Stricktly speaking, the spectral indices for $\delta N$ and $\delta H$ may be different but in fact it is too difficult to distinguish between these in the measured data. For the microscale magnetogasdynamic turbulence in the space plasma like that studied by Higdon (1986) the $11 / 3$ power is applicable. Let assume $\omega_{H}$ and $\omega_{p}$ are the gyro and the plasma frequency, respectively, $\omega$ is the radiation frequency, $z$ the plasma layer thickness and $c$ the speed of light. First, we consider the equations for the mean square deviations of the total flux density, $\sigma_{I}^{2}=\Gamma_{V}(0,0)$ and the circularly polarized component $\sigma_{V}^{2}=\Gamma_{V}(0,0)$. These can be represented as sums of contributions from fluctuations in the electron density, $\delta N$, and the magnetic field fluctuations, $\delta H$, viz.

$$
\sigma_{I, V^{2}}=\sigma_{I, V^{(N)^{2}}}+\sigma_{I, V}^{(H)^{2}}
$$

With $V_{0}^{2} \gg I_{0}^{2}\left(\omega / \omega_{H}\right)^{2}$ the equations for $\sigma_{I}^{(N)^{2}}$ and $\sigma_{V}^{(N)^{2}}$ are similar to one another and can be written as

$$
\left\{\begin{array}{l}
\sigma_{I}^{(N)^{2}} \\
\sigma_{V}^{(N)^{2}}
\end{array}\right\}=\left\{\begin{array}{l}
I_{0}^{2} \\
V_{0}^{2}
\end{array}\right\} 0.212 \frac{z^{11 / 6} \omega_{p}^{4}}{c^{7 / 6} \omega^{17 / 6}}
$$

In the case when the circularly polarized component $V_{0}$ is absent in the initial flux from the source,or its fraction is $V_{0}{ }^{2} \ll I_{0}{ }^{2}\left(\omega_{H} / \omega\right)^{2}$, we can write

$$
\sigma_{V}^{(N)^{2}}=\sigma_{I}^{(N)^{2}}\left(\omega_{H} / \omega\right)^{2}
$$

Here $\sigma_{I}^{(N)^{2}}$ is determined by Eq. (5). The second term in Eq.(4) owing to scattering from longitudional inhomogeneities of the magnetic field is

$$
\left\{\begin{array}{c}
\sigma_{I}^{(H)^{2}} \\
\sigma_{V}^{(H)^{2}}
\end{array}\right\}=\left\{\begin{array}{c}
V_{0}^{2} \\
I_{0}^{2}
\end{array}\right\} 0.212 \frac{z^{11 / 6} \omega_{p}^{4} \omega_{H}^{2}}{c^{7 / 6} \omega^{29 / 6}} C_{H}{ }^{2}
$$


for $V_{0}^{2} \gg I_{0}^{2}\left(\omega_{H} / \omega\right)^{2}$ or

$$
\sigma_{I}^{(H)^{2}}=4\left(\omega_{H} / \omega\right)^{2} \sigma_{V}^{(H)^{2}}
$$

for $V_{0}^{2} \ll I_{0}^{2}\left(\omega_{H} / \omega\right)^{2}$ and $\sigma_{V}^{(H)^{2}}$ as given by Eq. (7).

Analysing the mean-square fluctuations of the linearly polarized component we can distinguish two limiting cases, depending on the value of the mean-square phase difference of the ordinary and extraordinary wave,

$$
\gamma_{T}^{2}=0.365 \frac{z \omega_{p}^{4} \omega_{H}^{2}}{c^{2} \omega^{2}}\left[C_{N}^{2} L_{0 N}^{5 / 3}+C_{H}^{2} L_{0 H}^{5 / 3}\right]
$$

where $L_{0 H}$ and $L_{0 N}$ are the outer turbulence scales for the $\delta N$ and $\delta H$ fluctuations. With $\gamma_{T}{ }^{2} \ll 1$, we have for the mean squared deviation of the linear polarization, $\sigma_{L}^{2}=\sigma_{Q}{ }^{2}+\sigma_{U}{ }^{2}$

$$
\sigma_{L}{ }^{2} \approx\left[Q_{0}{ }^{2}+U_{0}{ }^{2}\right]\left\{4 \gamma_{T}{ }^{2}+0.212 \frac{z^{11 / 6} \omega_{p}^{4}}{c^{7 / 6} \omega^{17 / 6}}\left[C_{N}{ }^{2}-\left(\frac{\omega_{H}}{\omega}\right)^{2} C_{H}{ }^{2}\right]\right\}
$$

Here $Q_{0}, U_{0}$ and $\chi_{0}$ are the Stokes parameters and the position angle of the linearly polarized radiation near the source, and $\phi_{F}=\left(\omega_{p}^{2} \omega_{H} z\right) /(c \omega)^{2}$ is the Faraday rotation angle in plasma layer. With ${\gamma_{T}}^{2} \gg 1$, the mean-squared deviations in $Q$ and $U$ can be written as

$$
\sigma_{Q}^{2} \approx \sigma_{U}^{2}=\frac{1}{2}\left[Q_{0}^{2}+U_{0}^{2}\right]
$$

As can be seen from Eq.(9), the fluctuations in the linearly polarized component arise either from the phase, $\gamma_{T}{ }^{2}$, or the amplitude fluctuations. Note that the equations for linearly polarized fluctuations $\sigma_{Q}{ }^{2}$ and $\sigma_{U}{ }^{2}$ obtained by Spangler(1982) for the case of nonrelativistic plasma involved just a single term, $\boldsymbol{\gamma}_{T}{ }^{2}$, (cf. Eq.(10)) owing to phase difference of the fluctuations in the ordinary and extraordinary waves, The mean-square deviation of the measure of rotation, $\sigma_{R M}{ }^{2}$, is related to $\gamma_{T}{ }^{2}$ by means of the equation $\gamma_{T}{ }^{2}=\lambda^{4} \sigma_{R M}{ }^{2}$. In the other limiting case, i.e. $\gamma_{T}{ }^{2} \gg 1$, the mean-square deviation of the linear polarization fluctuations reaches a saturation level determined by the fraction of the linear polarization in the radiation from the source, $Q_{0}{ }^{2}+U_{0}{ }^{2}$. Thereby, the averaged Stokes parameters are exponentially small $\left(\langle Q\rangle \sim\langle U\rangle \sim \exp \left(-2 \gamma_{T}{ }^{2}\right)\right.$ ), since the interference of the ordinary and extraordinary waves is destroyed. As can be seen, the interference terms are not included in the invariant $\left\langle Q^{2}\right\rangle+\left\langle U^{2}\right\rangle$. Therefore, with $\gamma_{T}^{2} \gg 1$, the degree of linear polarization can be estimated from measurements of fourth-order field moments.

\section{References}

Higdon J.C. Astrophys. J.,1986,V. 310,p. 342.

Spangler S.R., Astrophys. J.,1982,V.261, p.310. 